\title{
Regulation of the innate immune system by autophagy: monocytes, macrophages, dendritic cells and antigen presentation
}

\author{
Nina Germic ${ }^{1} \cdot$ Ziva Frangez $^{1} \cdot$ Shida Yousefi ${ }^{1}{ }^{1} \cdot$ Hans-Uwe Simon $\mathbb{D}^{1,2}$
}

Received: 16 January 2019 / Revised: 19 January 2019 / Accepted: 22 January 2019 / Published online: 8 February 2019

(c) ADMC Associazione Differenziamento e Morte Cellulare 2019

\begin{abstract}
Autophagy is well equipped functionally to isolate microbial pathogens in autophagosomes and to carry out their clearance by dismemberment in the course of catabolic processes in the lysosome. Clearly, this is a non-metabolic function of autophagy that impacts strongly on the immune system. While in a preceding article on neutrophils, eosinophils, mast cells, and natural killer cells our focus was on the role of autophagy in regulating innate immune cell differentiation, degranulation, phagocytosis and extracellular trap formation, here we discuss monocytes/macrophages and dendritic cells, specifically, the influence of autophagy on functional cellular responses, such as phagocytosis, antigen presentation, cytokine production, control of inflammasome activation, tolerance and the consequences for overall host defense.
\end{abstract}

\section{Facts}

- The impact of autophagy on innate immunity extends beyond classical xenophagy, including extensive crosstalk with other host defense mechanisms.

- Autophagy in macrophages has different functions, being responsible for xenophagy, LC3-associated phagocytosis (LAP), production and delivery of antimicrobial peptides, and control of inflammasome activation.

- Many pathogens have developed strategies to escape xenophagy and LAP.

- In dendritic cells (DCs), autophagy is involved in each step of functional maturation, such as antigen presentation, migration, and cytokine secretion.

Edited by G. Melino

Hans-Uwe Simon

hus@pki.unibe.ch

Institute of Pharmacology, University of Bern, Bern, Switzerland

2 Department of Clinical Immunology and Allergology, Sechenov University, Moscow, Russia

\section{Open questions}

- The role of autophagy for the development of tolerance has remained poorly investigated.

- The immune regulatory effects mediated by autophagy need to be further elucidated.

- Can we translate current knowledge to improve the efficacy of antigen-specific immunotherapies and/or to develop novel anti-inflammatory drugs?

\section{Introduction}

As summarized in our preceding article and described in detail in excellent reviews, autophagy is a catabolic process responsible for turnover of macromolecules and organelles through lysosomal degradation [1-3]. With this process, autophagy satisfies multiple requirements. Turnover of macromolecules not only removes unwanted, malfunctioning or potentially harmful materials and structures; it also provides for replenishing metabolic intermediates, and, in addition, some intermediate breakdown products supply also materials like antimicrobial peptides and antigenic fragments for presentation by innate immune cells. In mammals, autophagy is regulated by the mammalian target of rapamycin (mTOR) and is carried out by autophagy-related (ATG) proteins. Identification of $A T G$ genes has provided the impetus for a molecular understanding of autophagy [2, 3]. Autophagy functions have been evolutionarily conserved 
from Saccharomyces to man and there are now known more than 40 functionally characterized $A T G$ genes which are essential for canonical autophagy. Canonical autophagic pathways were summarized in the preceding review on the role of autophagy in the innate immune cells [1]. Additional, non-canonical pathways that resemble autophagy or use some ATG proteins, but not others, have been characterized in excellent reviews elsewhere [4-6].

Monocytes are leukocytes that can differentiate into macrophages and myeloid lineage dendritic cells. Monocytes and their macrophage and dendritic-cell progeny serve three main functions in the immune system: phagocytosis, antigen presentation and cytokine production. After leaving the bone marrow, monocytes circulate in the bloodstream for about 1-3 days, moving then into tissues throughout the body where they undergo differentiation into macrophages and dendritic cells. They constitute between $4-10 \%$ of the leukocytes in the blood [7,8]. Monocytes are important players during inflammation and following pathogen challenge. Tissue-resident macrophages, on the other hand, have important functions during development and for tissue homeostasis, or for the resolution of an inflammation [9].

Dendritic cells (DCs) are professional antigen-presenting cells, though other leukocytes, B cells and macrophages, also present antigens. DCs are specific antigen-presenting cells with significant and potent functional plasticity that can act as intermediators between innate and adaptive immune responses. Although present at low density, due to their ubiquitous distribution in the body they are able to serve as sensors for danger signals and to mediate protective immunity or tolerance to self [10]. There are two distinct types of DCs with diverse functions. In the mid-1990s, in murine lymphoid organs two subsets of DCs was defined based on the presence or absence of CD8 expression, exhibiting distinct immune functions. Over a long period, these findings have been extended to DCs in nonlymphoid tissues. More recently, with the identification of a population of cells closely resembling plasma cells, but, upon exposure to viral stimuli, able to produce high levels of IFN- $\alpha$, a further major division in the DC family has been recognized. These cells, called plasmacytoid DCs (pDCs), can differentiate into immunogenic DCs that, upon stimulation, prime $\mathrm{T}$ cells against viral antigens. To distinguish pDCs from the DCs described earlier, the latter have been renamed "classical" DCs (cDCs) [11].

Autophagy contributes to cell survival in an important way by maintaining a metabolic balance, but also by performing essential quality control on organelles and removal of unneeded and aggregated macromolecules. Thus, as in almost all cells, it is not surprising that autophagy impacts on the immune system. However, the autophagic facility for packaging, dismemberment and removal of unwanted materials provides special opportunities for antimicrobial defense. Consequently, the role of autophagy in regulating innate immune cells is important and is under intense investigation. In an accompanying article published in this issue, we focussed on functions of autophagy which regulate the behavior of neutrophils, eosinophils, mast cells and natural killer (NK) cells [1]. In the present review, we summarize what is known about autophagy as impacting on the functions performed by monocytes/macrophages and DCs.

\section{Role of autophagy in monocytes/ macrophages}

\section{Differentiation}

It has been reported that autophagy is induced and is required for proper macrophage differentiation of human and murine monocytes driven by colony-stimulating factor1 (CSF-1) or granulocyte/macrophage colony-stimulating factor (GM-CSF) [12]. The induction of autophagy has been shown to be essential for survival and differentiation of monocytes. The differentiation signal releases BCL-2interacting coiled-coil protein 1 (Beclin 1) from BCL-2 by activating c-Jun N-terminal kinase (JNK) and preventing ATG5 cleavage, both of which are pivotal for the autophagy initiation [13]. Preventing the induction of autophagy hinders differentiation and cytokine production; thus, autophagy is important in the transition from monocyte apoptosis to differentiation [13]. Collectively, these findings point to an essential role for autophagy in monocyte differentiation (Fig. 1).

\section{Bacterial clearance}

Both autophagy and phagocytosis represent evolutionarily conserved eukaryotic mechanisms for engulfment and disposal of unwanted, and potentially harmful, materials. However, in contrast to phagocytosis, autophagy can also dispose of cytoplasmic materials by engulfing them in the double membrane vesicles called autophagosomes. Phagocytosis is the main mechanism in the repertoire of the innate immune system for eliminating bacteria. These are targeted into phagosomes in which they are then delivered to the lysosome for degradation [14]. Some bacteria, however, are able to escape the phagosome and to multiply in the cytosol. In another approach, some bacteria modify the phagosome to prevent its fusion with the lysosome [15]. In such cases, autophagy presents an alternative mechanism that can target bacteria into autophagosomes which subsequently fuse successfully with the lysosome [16-19] (Fig. 2).

How is this process of defense autophagy initiated? It seems likely that the triggers are produced by the bacteria 


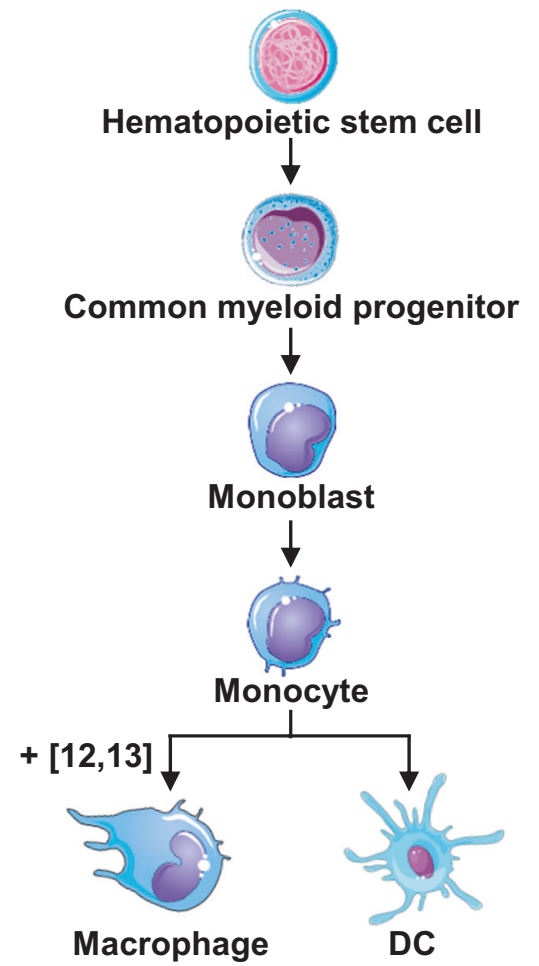

Fig. 1 Autophagic activity in the differentiation from monocytes to macrophages. Innate immune cells such as macrophages and DCs arise from a hematopoietic stem cell (HSC) that has committed to a common myeloid progenitor (CMP). Monoblasts then further generate a monocytic cell lineage, leading to the development of macrophages and DCs. To date, autophagy is known to be involved in the differentiation step from monocytes to macrophages. Numbers in brackets designate references: + , enhanced function

themselves; that Toll-like receptors (TLRs) might play a role has been suggested [20-22]. For instance, TLR4 activation results in the recruitment of the adaptors myeloid differentiation primary response 88 (MyD88) and Toll/IL-1 receptor (TIR) domain-containing adaptor inducing interferon $\beta$ (TRIF), which can bind Beclin 1 to initiate autophagy in mouse macrophage RAW264.7 cells [23]. In addition, it has been reported that tumor necrosis factor (TNF) receptor-associated factor 6 (TRAF6)-mediated, Lys $^{63}$ (K63)-linked ubiquitination of Beclin 1 is essential for TLR4-triggered autophagy in macrophages. Two TRAF6-binding motifs in Beclin 1 facilitate TRAF6binding and Beclin 1 ubiquitination. Thus, the degree of K63-linked Beclin 1 ubiquitination plays a key role in regulating autophagy [24]. Moreover, neutrophils release extracellular vesicles (EVs) to the extracellular milieu that then participate in intercellular communication. Ligands for TLR-2/6 were detected in EVs released from $M$. tuberculosis-infected neutrophils (EV-TB). Such EVs induced elevated expression of microtubule-associated proteins $1 \mathrm{~A} /$ 1B light chains (LC3-II) in macrophages and LC3-II colocalization with bacteria present inside infected macrophages. The intracellular mycobacterial load increased after wortmannin inhibition of autophagy. In conclusion, EV-TB promotes macrophage autophagy and thereby reduces intracellular mycobacterial survival [25].

Another possibility is that pathogens trigger cytosolic receptors, which subsequently induce autophagy. Consistent with this concept, both nucleotide-binding oligomerization domain (NOD)1 and NOD2 activation led to an upregulation of autophagy after ATG16L1 was recruited to bacterial invasion sites [26]. Since cytokines, such as tumor necrosis factor (TNF)- $\alpha$ [27], TNF-related apoptosis-inducing ligand (TRAIL) [28], and interferon (IFN)- $\gamma$ [17] also increase autophagic activity, TLR-mediated targeting of intracellular pathogens might not be direct, but rather involve cytokines. It should be noted that interleukin (IL) -10 and IL-10 receptor signaling inhibits autophagy induction in murine macrophages. The mechanism involves the activation of the class I phosphatidylinositol 3-kinase (PI3K)-pathway, which leads to the phosphorylation of the 70-kilodalton ( $\mathrm{kDa}$ ) ribosomal protein S6 kinase (p70S6K) through the activation of AKT/PKB (protein kinase B) and the mammalian target of rapamycin complex 1 (mTORC1) [29]. Besides cytokines, vitamin D3 is believed to modulate autophagic activity in human macrophages via cathelicidin, which activates transcription of Beclin 1 and ATG5. Colocalization of mycobacterial phagosomes with autophagosomes was also induced by vitamin D3 in a cathelicidin-dependent manner [30].

Autophagy was induced after CD40 activation of macrophages, helping to fuse Toxoplasma gondii (T. gondii)containing vacuoles with lysosomes, resulting in killing of the pathogen [31]. In addition to the initiation of autophagy and maturation of autophagosomes, p62 was required for mycobactericidal activity. p62 was responsible for delivery of specific ribosomal and bulk ubiquitinated cytosolic proteins to autolysosomes where they underwent proteolytic processing into products capable of killing Mycobacterium (M.) tuberculosis, indicating some of the unique bactericidal properties of autophagic organelles [32].

Interesting strategies for escape from lysosomal killing mechanisms have been developed by many pathogens, for example by blocking the fusion of phagosomes with lysosomes, demonstrating the importance of autophagy induction for pathogen killing by monocytes/macrophages [33] (Fig. 2).

\section{Phagocytosis}

Autophagy has also been associated with innate immunity against pathogens even where pathogens have developed no phagosome escape mechanisms. In such cases, autophagy seems able to enhance the fusion of phagosomes with lysosomes, seemingly without the intermediate formation of 


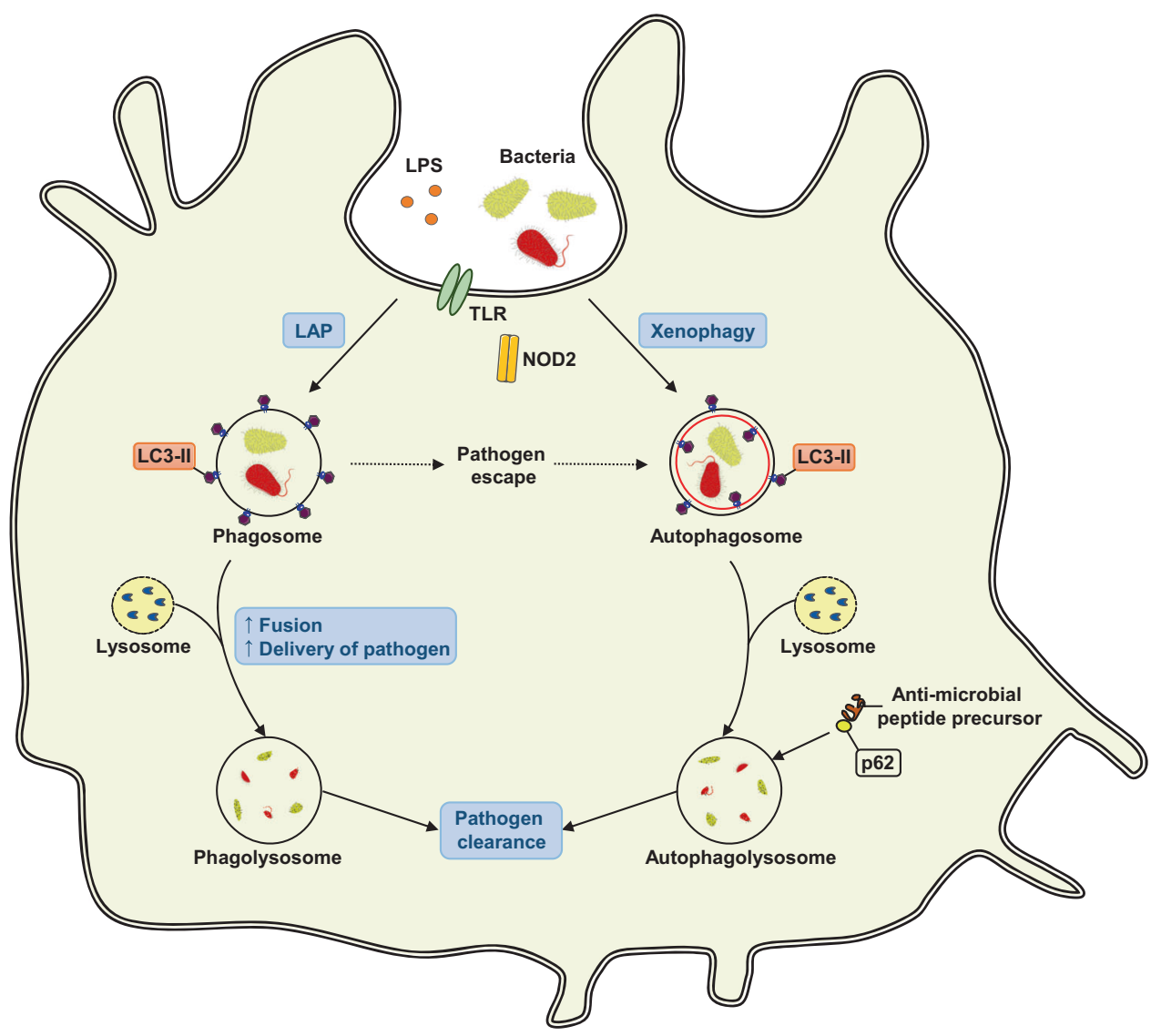

Fig. 2 Involvement of autophagy in the process of phagocytosis. Autophagy in phagocytic cells is regulated by a variety of immunological signals. Pathogens on the cell surface or in the cytosol are recognized by pattern-recognition receptors, such as Toll-like receptors or Nod-like receptors. The most direct role of autophagy for the removal of microbes is a selective engulfment of intracellular pathogens by the autophagosomal membrane and their subsequent

autophagosomes [20]. The result is a rapid destruction of the engulfed pathogen. Prior to fusion, phagosomes recruit LC3 in a process requiring ATG5, Beclin 1, ATG7 and PI3K activity [20] together with TLR signaling in order to recognize that the phagosome contains a pathogen [33]. On the other hand, macrophages lacking ATG7 demonstrated increased bacterial uptake when infected with $M$. tuberculosis or with M. tuberculosis var. bovis BCG. Atg $7^{-/-}$macrophages showed elevated expression of two class A scavenger receptors: the macrophage receptor with collagenous structure (MARCO) and the macrophage scavenger receptor 1 (MSR1). This increase in scavenger receptors resulted from an increased nuclear factor (erythroid-derived 2)-like 2 (NFE2L2) transcription factor activity as a consequence of accumulating p62 in $\operatorname{Atg} 7^{-1-}$ macrophages [34].

M. tuberculosis is also known as an intracellular pathogen persisting within phagosomes owing to its interference with phagolysosome biogenesis. Stimulation of autophagic degradation after fusion with the lysosome, a process called xenophagy. Protein p62 can deliver specific ubiquitinated proteins to autophagolysosomes where they are converted into microbial killing products. Another autophagic contribution to pathogen removal is through LC3-associated phagocytosis (LAP). LC3-II binds to the phagosomal membrane, facilitating phagosomal maturation and its fusion with the lysosome to enhance pathogen removal

pathways in macrophages increases the maturation of mycobacterial phagosomes into phagolysosomes. Stimulation of autophagy physiologically, or pharmacologically with rapamycin, caused mycobacterial phagosome colocalization with LC3. Hence, that autophagic pathways can overcome the $M$. tuberculosis trafficking block is apparent from these findings [17].

The promotion of phagocytosis by autophagy has also been demonstrated in other models. Adenosine triphosphate (ATP) treatment of M. tuberculosis var. bovis BCG-infected human macrophages induced a $\mathrm{Ca}^{2+}$ and $\mathrm{P}_{2} \mathrm{X}_{7}$ (purinoceptors for ATP) receptor-dependent killing of intracellular mycobacteria which required autophagic activity. The bacteria were demonstrated within the characteristic doublemembraned autophagosomes of ATP-treated, BCG-infected monocyte-derived macrophages. Autophagy inhibition by wortmannin or pre-treatment of macrophages with anti$\mathrm{P}_{2} \mathrm{X}_{7}$ antibody blocked ATP-induced phago-lysosomal fusion. Stimulation of autophagy with ATP was also 
temporally associated with a drop in intracellular mycobacterial viability, but this could be suppressed by treatment with wortmannin or the selective $\mathrm{P}_{2} \mathrm{X}_{7}$ antagonist, oxidized ATP. Collectively, ATP/P2 $X_{7}$-mediated killing of intracellular mycobacteria was shown to be dependent on the induction of autophagy [35].

Another study reported a requirement for ATG5 for in vivo resistance to the intracellular pathogens Listeria monocytogenes (L. monocytogenes) and T. gondii. In primary macrophages, ATG5 was required for IFN- $\gamma / \mathrm{LPS}-$ induced damage to the $T$. gondii parasitophorous vacuole membrane and for parasite clearance. While no classical hallmarks of autophagy were detected, i.e. autophagosomes enveloping $T$. gondii, ATG5 was presumably required to recruit the IFN- $\gamma$-inducible p47 GTPase IIGP1 (Irga6) to the vacuole membrane, an event which is responsible for the clearance of $T$. gondii [36]. This work established that ATG5 expression in phagocytic cells is essential for cellular immunity to intracellular pathogens in vivo. Furthermore, it was a demonstration that an autophagy protein can participate in immunity and intracellular killing of pathogens through autophagosome-independent processes such as GTPase trafficking.

Autophagy, whether induced by physiological or pharmacological means, reduced the phagocytic capacity of murine macrophages, regardless of the source of the cells or the nature of the particles being phagocytosed. The inhibitory effect of autophagy on phagocytosis was early and reversible, resulting in no loss of cell viability. Furthermore, the induction of autophagy did not affect the capacity to recognize and bind particles, indicating that autophagy does not inhibit the particle recognition process in the macrophage, even though particle internalization is suppressed [37]. Moreover, a different process called LC3-associated phagocytosis (LAP), has been described recently. The molecular requirements for LAP are at least partially different from canonical autophagy [20, 38, 39]. Hence, these findings support the notion that phagocytosis and autophagy can be interdependent and complementary processes (Fig. 2).

\section{Cytokine production}

Blocking autophagy enhances IL-1 $\beta$ production in monocytes/macrophages. Loss of autophagy due to ATG16L1 deficiency increases IL-1 $\beta$ and IL-18 levels in a mouse model of gut inflammation, suggesting that autophagy controls inflammasome activation and limits production of IL-1 $\beta$ and IL-18 (ref. [40]). Similarly, a lack of ATG7 resulted in elevated production of IL-1 $\beta$ and pyroptosis, thus consistent with enhanced inflammasome activation [41]. Moreover, it has been reported that ATG5 suppresses the production of IL-1 $\beta$ [42].
Similarly, depletion of LC3 or Beclin 1 caused increased activation of caspase- 1 and secretion of IL- $1 \beta$ and IL-18. In addition, any lack of autophagic proteins in macrophages increased the accumulation of damaged or dysfunctional mitochondria and the cytosolic translocation of mitochondrial DNA (mtDNA) in response to lipopolysaccharide (LPS) and ATP treatment. Release of mtDNA into the cytosol depended on the NACHT, LRR and PYD domainscontaining protein 3 (NALP3, also called NLRP3) inflammasome and availability of mitochondrial reactive oxygen species (ROS). Cytosolic mtDNA contributed to the secretion of IL-1 $\beta$ and IL-18 in response to LPS and ATP [43]. Therefore, this study implied that autophagic proteins regulate a (NOD)-like receptor protein 3 (NLRP3), and that prevention of dysfunctional mitochondrial removal via autophagy/mitophagy causes NLRP3-dependent inflammation [43]. In agreement with this assumption, it was reported that the NLRP3 inflammasome can sense mitochondrial dysfunction, thus explaining the frequent accumulation of damaged mitochondria in inflammatory diseases [44]. It was subsequently shown that autophagy degrades inflammasome components, while processed caspase-1 is reduced in cell extracts following stimulation of autophagy [45] (Fig. 3).

After treatment of macrophages with TLR ligands, proIL-1 $\beta$ was specifically sequestered into autophagosomes. Further autophagy activation using rapamycin induced pro-IL-1 $\beta$ degradation, blocking secretion of the mature cytokine. Inhibition of autophagy promoted processing and secretion of IL-1 $\beta$ by antigen-presenting cells in an NLRP3- and TRIF-dependent manner. Autophagy induction in mice in vivo using rapamycin diminished serum IL- $1 \beta$ levels following challenge with LPS. Thus, autophagy controls production of IL- $1 \beta$ in two ways: by targeting lysosomal degradation of pro-IL-1 $\beta$ and by regulating the activation of the NLRP3 inflammasome [46] (Fig. 3).

The link between autophagy and cytokine production plays a role in multiple chronic inflammatory diseases. For instance, Gaucher disease is an inherited deficiency in lysosomal glucocerebrosidase, with increased secretion of inflammatory cytokines. Studying macrophages of these patients revealed increased inflammasome activation as a result of impaired autophagy. The small-molecule glucocerebrosidase chaperone NCGC758 was able to reverse the defects in parallel with autophagy induction and lowered IL-1 $\beta$ secretion, underlining the role of lysosomal glucocerebrosidase deficiency in these processes. Moreover, in Gaucher macrophages, increased levels of the autophagic adaptor p62 prevented incorporation of inflammasomes into autophagosomes. Increased p62 levels caused activation of p65-NF- $\mathrm{KB}$ in the nucleus, promoting inflammatory cytokine expression and secretion of IL-1 $\beta$. This novel 


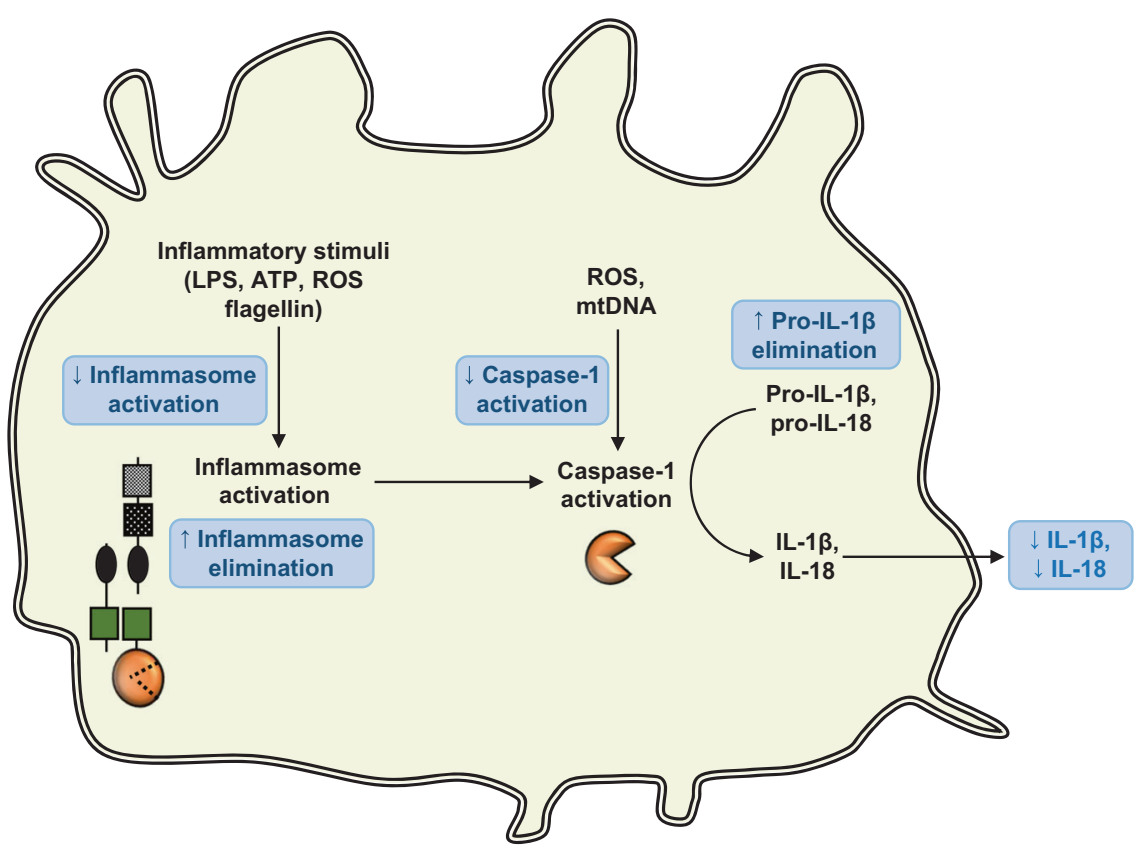

Fig. 3 Regulation of inflammasomes by autophagy. Autophagy negatively controls the activation of inflammasomes in response to inflammatory stimuli and degrades inflammasome components, leading to reduced caspase- 1 activation. Decreased caspase- 1 activation blocks conversion of the inactive cytokine precursors pro-IL-1 $\beta$ and pro-IL-18 to active IL- $1 \beta$ and IL-18 forms, which would otherwise be released from the cell to perform proinflammatory functions.

mechanism connects the idea of lysosomal dysfunction with inflammasome activation [47].

Furthermore, deletion of several autophagy genes in macrophages can cause an inflammation-mediated eye disease, uveitis, and can lead to blindness. A failure in autophagy activates inflammasome-mediated IL-1 $\beta$ secretion, thereby increasing disease severity. Interestingly, experimental uveitis was also found to be elevated in a model for Crohn disease, a systemic autoimmune disease in which patients often develop uveitis. This finding provides a potential mechanistic link between macrophage autophagy and systemic disease [48]. Moreover, macrophages isolated from human diabetic wounds exhibited a proinflammatory phenotype, including sustained NLRP3 inflammasome activity associated with IL- $1 \beta$ secretion. Autophagy inhibition plays an important role in this process [49] (Fig. 3).

Macrophages and neutrophils of mice with a LysM-cremediated conditional knockout of Atg 5 developed systemic and hepatic inflammation following treatment with lowdose LPS and a high-fat diet. Loss of autophagy promoted inflammation through the regulation of macrophage polarization. Bone marrow-derived macrophages and Kupffer cells from knockout mice exhibited abnormalities in polarization with both increased proinflammatory M1 and decreased anti-inflammatory $\mathrm{M} 2$ polarization as determined by measurements on genes and proteins [50].
Autophagy also directly regulates IL- $1 \beta$ production by targeting proIL-1 $\beta$ for lysosomal degradation. In the absence of autophagy, dysfunctional mitochondria accumulate in the cells, causing the release of mtDNA in the cytosol and increasing intracellular ROS levels. As a consequence, the activation of the inflammasome and caspase- 1 is accelerated, leading to an increased secretion of IL- $1 \beta$ and IL-18

Moreover, autophagy inhibition was accompanied by a shift to aerobic glycolysis together with a lowered mitochondrial membrane potential (MOMP). The reduced MOMP combined with autophagy inhibition resulted in an increased mitochondrial content and elevated cellular ROS levels which eventually led to cell death. In human macrophages, AKT/PKB was identified as being responsible for the glycolytic shift and autophagy inhibition. Surprisingly, AKT/PKB knockdown rescued these cells from death. In agreement with these observations, inflammatory M1 macrophages with active glycolysis exhibit reduced autophagy [51]. Furthermore, macrophages from aged mice demonstrated a definitely reduced autophagic flux compared to macrophages from young mice. The reduced autophagy skews macrophage metabolism toward glycolysis. Therefore, a modulation of autophagy may allow us to control excess inflammation and to maintain macrophage function during aging [52].

Taken together, one can say that autophagy somehow limits inflammatory responses. Pharmacological inhibition of mTOR can thus act to produce increased antiinflammatory activities, also in humans [53]. Clearly, further experimental work and clinical trials will be necessary to reach a better understanding of the the potential link between autophagy and the intensity of innate immune responses. 


\section{Role of autophagy in dendritic cells}

\section{Antigen presentation by MHC class II molecules}

Lysosomal degradation products are known to be presented via major histocompatibility complex (MHC) class II molecules to $\mathrm{CD}^{+} \mathrm{T}$ cells and it is these helper $\mathrm{T}$ cells which orchestrate the specific immune response [54]. Interestingly, this pathway may also be responsible for antigen presentation by other cells expressing MHC class II molecules, even those lacking a high endocytic capacity, such as, for example, cortical thymic epithelial cells [55]. As discussed above, autophagy is used to direct pathogens into autophagosomes subsequently fusing with lysosomes [16-19]. In this way, epitopes of pathogens and intracellular antigens can be delivered to MHC class II molecules [5659]. It is therefore unsurprising that autophagic pathways promote MHC class II antigen-presentation. Thus, apart from its role in innate immunity, autophagy may also serve to alarm the adaptive immune system against pathogens that were delivered in DCs by the autophagy pathway for lysosomal degradation. In the following, we provide some examples how autophagy connects DCs with adaptive immunity.

It has been reported that 3-methyladenine (3-MA) decreased MHC class II presentation of an endogenously expressed bacterial peptide [56]. Autophagy induction upon amino-acid starvation promoted MHC-II presentation of peptides derived from both intracellular and lysosomal source proteins [57]. Pharmacological and genetic inhibition of autophagy diminished efficient MHC class II presentation of an endogenously synthesized viral protein (Epstein-Barr virus nuclear antigen 1 (EBNA1)) [58]. The initial studies led to the generally accepted concept that the autophagy pathway facilitates transfer of cytosolic antigens directly to late endosomal or lysosomal compartments, as opposed to the processing of exogenous antigens captured through endocytosis or phagocytosis in antigen-presenting cells [59].

Autophagosomes continuously fuse with multivesicular MHC class II-loading compartments. This pathway is of functional relevance, because targeting of influenza matrix protein 1 to autophagosomes via its fusion with the autophagosome-associated protein LC3 caused strongly enhanced MHC class II presentation to $\mathrm{CD} 4^{+} \mathrm{T}$ cell clones. Therefore, autophagy constitutively and efficiently delivers cytosolic proteins for MHC class II presentation and this facility can be utilized for improved helper $\mathrm{T}$ cell stimulation $[60,61]$. This pathway is also of in vivo relevance, since mice with a DC-conditional deletion in Atg5 showed impaired $\mathrm{CD}^{+}{ }^{+} \mathrm{T}$ cell priming after Herpes simplex virus infection and succumbed rapidly to the disease. The most pronounced defect in $\mathrm{Atg} 5^{-/}$DCs was with phagosome-to- lysosome fusion and subsequent processing and presentation of phagocytized antigens containing TLR stimuli for MHC class II. In contrast, cross-presentation of peptides on MHC class I was unaffected by the absence of Atg5 (ref. [62]) (Fig. 4).

Another report suggested that a subset of phagosomes becomes coated with LC3 and maintains phagocytized antigens, allowing prolonged presentation on MHC class II molecules. Such LC3-positive phagosomes are formed around the antigen with TLR2 agonists and are dependent on ROS production by NADPH oxidase 2 (NOX2) for their generation. LC3-positive phagosomes do not contain other classical autophagy markers, showing delayed maturation and enabling prolonged antigen presentation by MHC class II molecules. A deficiency in the formation of these antigen storage phagosomes could impair $\mathrm{CD}^{+} \mathrm{T}$ cell responses and compromise immune control. Therefore, autophagy proteins stabilize pathogen-containing phagosomes for prolonged MHC-II antigen processing [63] (Fig. 4).

Dectin-1 is a pattern-recognition receptor that binds to $\beta$ glucans in fungal cell walls triggering phagocytosis and production of ROS by the NADPH oxidase, subsequently, together with inflammatory cytokines. It has been demonstrated that dectin-1 signaling in macrophages and bone marrow-derived dendritic cells (BMDC) triggers induction of autophagy. Furthermore, dectin-1 directs the recruitment of LC3-II to phagosomes, and for this, requires Syk, activation of ROS production by the NADPH oxidase, and ATG5. Employing LC3-deficient DCs, the authors showed that whereas LC3 recruitment to phagosomes is not important for triggering phagocytosis, microbial killing or dectin-1-mediated inflammatory cytokine production, its presence does facilitate recruitment of MHC class II molecules to phagosomes and enhances the presentation of fungal-derived antigens to $\mathrm{CD}^{+}{ }^{+} \mathrm{T}$ cells. Collectively, dectin-1-triggered recruitment of LC3 protein to phagosomes is fundamental for the MHC class II presentation of fungal-derived antigens [64].

NOD2 acts as a bacterial sensor in monocyte-derived DCs, but how does bacterial recognition link with antigen presentation after NOD2 stimulation? It has been shown that NOD2 triggering by muramyldipeptide induces autophagy in DCs. This effect requires receptor interacting protein kinase2 (RIPK-2), ATG5, ATG7 and ATG16L1. NOD2-mediated autophagy is necessary for bacterial processing and for eliciting MHC class II antigen-specific $\mathrm{CD}^{+}{ }^{+} \mathrm{T}$ cell responses in dendritic cells. DCs from individuals with Crohn's disease who express Crohn's disease-associated NOD2 or ATG16L1 risk variants, show defects in autophagy induction, bacterial trafficking and antigen presentation [65].

Antibody responses to citrullinated self-proteins are found in several autoimmunities, particularly in rheumatoid arthritis, where they serve as a diagnostic indicators. 


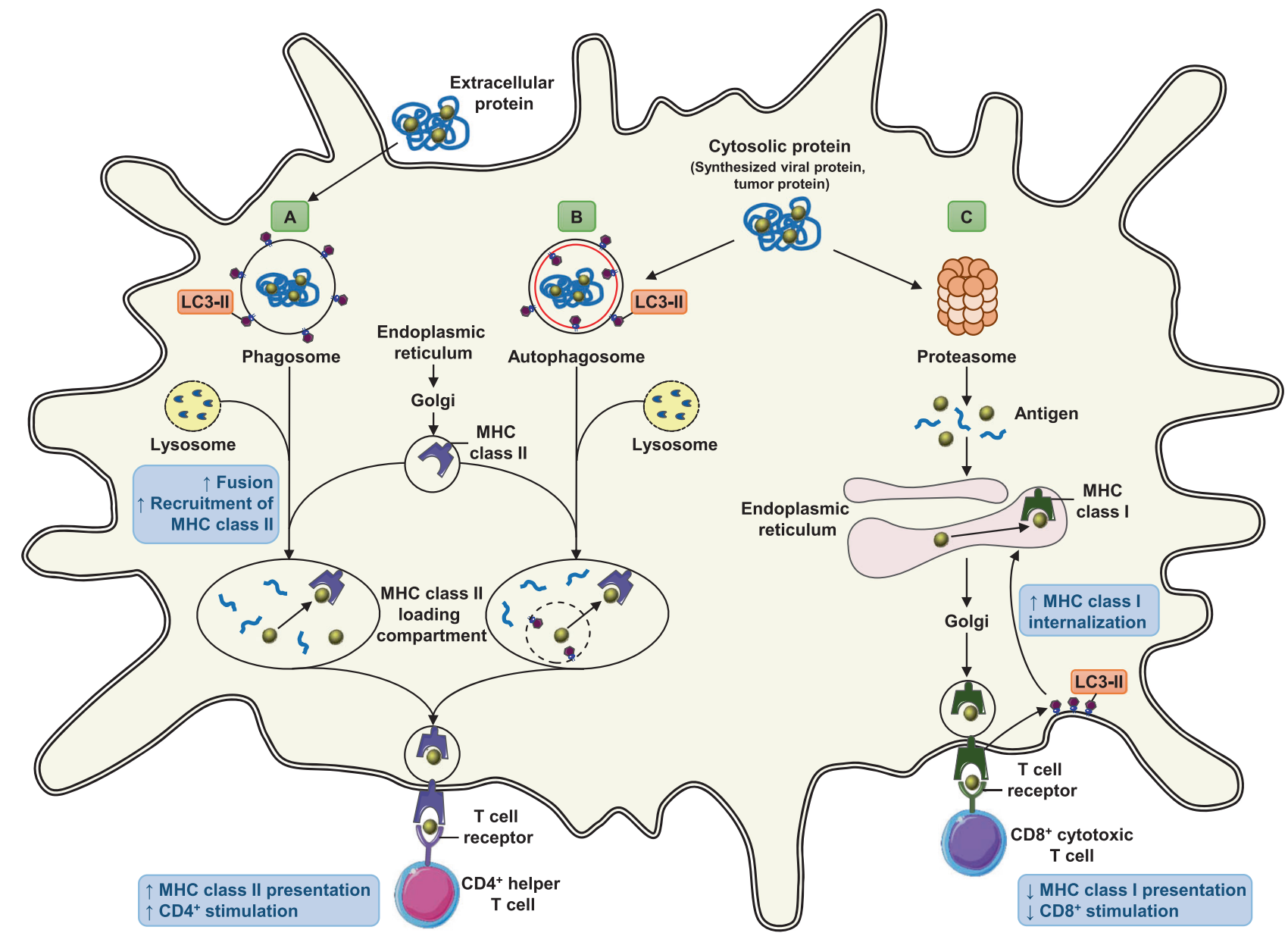

Fig. 4 Role of autophagy in antigen presentation by dendritic cells. Autophagy promotes the presentation of antigens on MHC class II molecules, but hinders their presentation on MHC class I molecules. a Autophagy supports presentation of exogenous phagocytosed antigens on MHC class II molecules. Extracellular proteins are phagocytosed, and LC3-II is recruited to phagosomal membranes enhancing fusion between phagosomes and lysosomes. Recruitment of MHC class II molecules to phagolysosomes and their loading with antigenic peptides is facilitated, leading to upregulation of MHC class II molecules on the surface of DCs and stimulation of $\mathrm{CD} 4^{+}$helper $\mathrm{T}$ cells. $\mathbf{b}$

Investigations have demonstrated that processing of the protein hen egg-white lysozyme (HEL) resulted in citrullination of peptides presented with MHC class II molecules of antigen-presenting cells. The presentation of these citrullinated peptides, but not of unmodified peptides, is a consequence and a biochemical marker for an autophagic response. DCs, macrophages, and thymic DCs presented citrullinated peptides constitutively. 3-MA treatment was highly selective as it blocked only presentation of citrullinated HEL peptides, but did not affect unmodified peptide presentation. Autophagy in antigen-presenting cells is a key cellular event for the generation and presentation of citrullinated peptides to $\mathrm{CD} 4^{+} \mathrm{T}$ cells. These findings indicate a connection between autophagy and autoreactivity through the generation of this new citrullinated epitope [66].
Autophagosomes can target cytosolic proteins for lysosomal degradation, delivering them to the MHC class II loading compartment. This classical autophagy pathway enables MHC class II presentation of intracellular antigens and gives rise to a $\mathrm{CD} 4{ }^{+}$helper $\mathrm{T}$ cell response. c Autophagy compromises the presentation of antigens on MHC class I molecules through their increased internalization. LC3-II molecules recruit the internalization machinery to MHC class I molecules on the surface of DCs and target them for intracellular transport, reducing $\mathrm{CD}{ }^{+}$cytotoxic $\mathrm{T}$ cell responses

Clearly, inducing autophagy in DCs leading to enhanced immunogenicity is a strategy to increase the success of vaccination [67, 68]. Moreover, unraveling the role of autophagic antigen processing and presentation has implications not only for infectious diseases, but also for cancer, autoimmunity, allergy, and transplantation medicine. It should be noted that some pathogens have developed mechanisms that suppress autophagy and inhibit MHC class II-restricted antigen presentation [69].

\section{Antigen presentation by MHC class I molecules}

Unlike the supportive role of autophagy for $\mathrm{CD}^{+} \mathrm{T}$ cell stimulation, autophagy diminishes antigen presentation on MHC class I molecules during anti-viral $\mathrm{CD} 8^{+} \mathrm{T}$ cell 
responses by enhancing MHC class I internalization in dendritic cells. Atg5- and Atg7-knockout DCs have elevated surface MHC class I levels owing to decreased endocytosis and degradation of MHC-I molecules. Therefore, with reduced ATG-dependent MHC class I internalization, DCs can stimulate $\mathrm{CD} 8^{+} \mathrm{T}$ cell responses more efficiently both in vitro and in vivo. During viral infections, lack of ATG5 results in enhanced influenza- and lymphocytic choriomeningitis virus (LCMV)-specific $\mathrm{CD} 8^{+} \mathrm{T}$ cell responses in vivo. Elevated influenza-specific $\mathrm{CD} 8^{+} \mathrm{T}$-cell responses go together with an improved immune control of this infection [70] (Fig. 4).

The endogenous presentation of most viral epitopes through the MHC class I pathway is entirely dependent on the transporter associated with the antigen processing (TAP) complex, which transfers the peptide products after proteasomal degradation into the endoplasmic reticulum. However, a pathway involving autophagy has been described; it could be shown that autophagy can mediate TAP-independent presentation of viral epitopes through a MHC class I pathway [71]. In addition, a recent publication shows an increase in the capacity of murine DCs to present viral antigens with MHC class I after infection with a mutant of Herpes simplex virus 1 (HSV-1- 434.5 ) which lacks infected cell protein 34.5 (ICP34.5), as compared to the wild-type HSV-1 strain. The ICP34.5 protein overcomes host cell translational arrest, suppressing autophagy. The lack of this protein leads to a low viral protein abundance, which, however, could be processed and presented efficiently, demonstrating the important role of autophagy in processing endogenous viral proteins in HSV-1-infected DCs [72].

\section{Cytokine production}

Plasmacytoid DCs (pDCs) are crucial mediators for the establishment of immunity against most viruses, given their extraordinary capacity to produce large quantities of type I IFNs. pDCs are able to detect viruses in acidified endosomes with the help of TLRs. Several studies presented evidence that the recognition of single-stranded RNA (ssRNA) viruses occurs via TLR7 and requires the transport of cytosolic viral replication intermediates into the lysosome by the process of autophagy. In this context, autophagy was found to be required for the production of IFN- $\alpha$ by pDCs [73]. Similarly, Simian Virus 5 (SV5) activated TLR7 in pDCs, resulting in the secretion of large amounts of IFN- $\alpha$ which depended on autophagy [74]. Moreover, large amounts of type I IFNs were released from isolated pDCs after exposure to Epstein-Barr virus (EBV) by a mechanism requiring both TLRs and the autophagic machinery. It should be noted, however, that EBV is able to induce activation, but impairs maturation of pDCs.
Therefore, TNF- $\alpha$ release is reduced and EBV-infected pDCs are unable to mount a full T-cell response, suggesting an immune-evasion strategy promoted by the virus [75].

Autophagy-dependent cytokine production was also observed in bone marrow-derived DCs (BMDC). After infection with the influenza A (H1N1) in autophagydeficient haploinsufficient Beclin $1^{+/-}$BMDCs, lower levels of IL- 6 , TNF- $\alpha$, IFN- $\beta$, IL-12p70 and IFN- $\gamma$ were secreted as compared to control BMDCs. H1N1-infected Beclin $1^{+/-}$BMDCs exhibited decreased activation of

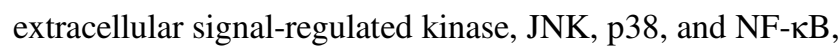
as well as IFN regulatory factor 7 nuclear translocation. H1N1-infected control BMDCs were more efficient in inducing allogeneic $\mathrm{CD} 4^{+} \mathrm{T}$-cell proliferation and driving $\mathrm{T}$ helper type 1, 2 and 17 cell differentiation while inhibiting $\mathrm{CD}^{+}$forkhead box $\mathrm{P}^{+}\left(\mathrm{Foxp}^{+}\right)$regulatory T-cell differentiation. These data indicate that autophagy is important in the regulation of BMDC immune responses to H1N1 infection [76]. Autophagy-dependent cytokine production after influenza A virus infection was also observed in other models. For instance, Ripk $2^{-1-}$ BMDCs exhibited defective autophagy of mitochondria (mitophagy), leading to enhanced mitochondrial superoxide production and consequent activation of the NLRP3 inflammasome with subsequent increased production of IL-18. RIPK2-regulated mitophagy is induced after phosphorylation and activation of the mitophagy inducer unc-51-like kinase 1 (ULK1). Accordingly, Ulk1 ${ }^{-1-}$ BMDCs exhibited also enhanced mitochondrial production of superoxide and activation of caspase-1. Taken together, these results demonstrate a role for NOD2-RIPK2 signaling in protection against virally triggered immunopathology by negatively regulating the activation of the NLRP3 inflammasome and production of IL-18 via the ULK1-dependent mitophagy in BMDCs [77].

Low levels of autophagy in immature monocyte-derived DCs (iDCs) were also associated with low cytokine production in the context of human immunodeficiency virus-1 (HIV-1) infection. Autophagy is blocked in iDCs, because the HIV-1 envelope protein activates the mTOR pathway. HIV-1-mediated downregulation of autophagy in DCs impaired both innate and adaptive immune responses [78].

Interestingly, defective autophagy in BMDCs can result in increased cytokine production by $\mathrm{T}$ cells. After a respiratory syncytial virus (RSV) infection in vivo, lungs of Beclin $1^{+/}$mice showed increased Th2 cytokine production, mucus secretion, and lung infiltration by eosinophils and inflammatory dendritic cells. Beclin $1^{+/-}$pulmonary and BMDCs showed decreased expression of MHC class II and innate cytokine production upon RSV infection. Further examination indicated that Beclin $1^{+/}$BMDCs stimulated less IFN- $\gamma$ and IL-17 production by cocultured $\mathrm{CD} 4^{+}$ $\mathrm{T}$ cells, but increased Th2 cytokine production in comparison with control BMDCs from wild-type mice [79]. 


\section{Tolerance}

Phagocytosis of apoptotic cells has been implicated in the maintenance of immune tolerance and prevention of excess inflammation. It has been reported that $\mathrm{T}$ cell immunoglobulin- and mucin domain protein-4 (TIM-4) repressed tumorspecific immunity which had been triggered by chemotherapy-induced tumor cell death. TIM-4 was highly expressed on tumor-associated myeloid cells such as macrophages and DCs. TIM-4 directly interacted with AMPdependent protein kinase $\alpha 1$ (AMPK $\alpha 1$ ) and activated autophagy-mediated degradation of ingested tumors, leading to reduced antigen presentation and impaired $\mathrm{CD}^{+}$cytotoxic T lymphocyte responses. Consistently, blockade of TIM-4AMPK $\alpha 1$-autophagy improved the antitumor effect of chemotherapeutics by enhancing tumor-specific $\mathrm{CD}^{+} \mathrm{T}$ cell immune responses. This finding provides insight into the immune tolerance mediated by phagocytosis of dying cells, and the concept of targeting the TIM-4-AMPK $\alpha 1$ interaction constitutes a unique strategy for augmenting antitumor immunity and improving cancer chemotherapy [80].

Moreover, it has been demonstrated that Foxp3 ${ }^{+} \mathrm{T}$ regulatory cells $\left(\mathrm{T}_{\text {regs }}\right)$ strongly inhibit autoimmune responses in vivo by blocking the autophagic machinery in DCs in a cytotoxic $\mathrm{T}$ lymphocyte-associated protein 4
(CTLA4)-dependent manner. Autophagy-deficient DCs exhibited reduced immunogenic potential and failed to prime autoantigen-specific $\mathrm{CD} 4^{+} \mathrm{T}$ cells to mediate autoimmunity. Mechanistically, CTLA4 binding promoted activation of the PI3K/AKT/mTOR axis and forkhead box $\mathrm{O}$ (FoxO)1 nuclear exclusion in DCs, leading to decreased transcription of the important autophagy component LC3. Human DCs treated with CTLA4-Ig, a fusion protein composed of the fragment crystallizable $(\mathrm{Fc})$ region of $\mathrm{IgG1}$ and the extracellular domain of CTLA4, elicited reduced levels of autophagosome formation, whereas DCs from CTLA4-Ig-treated rheumatoid arthritis patients showed reduced levels of LC3 transcripts. The canonical autophagy pathway in DCs was identified as a molecular target of Foxp3 ${ }^{+} \mathrm{T}_{\text {reg-mediated suppression that leads to }}$ amelioration of autoimmune responses [81] (Fig. 5).

Association of host DNA with autoantibodies activates TLR9, inducing the pathogenic secretion of type I IFNs from pDCs. In response to DNA-containing immune complexes, IFN- $\alpha$ production depends upon the convergence of phagocytic and autophagic pathways, a process called LC3associated phagocytosis (LAP; see above). LAP was required for TLR9 trafficking into a specialized IFN signaling compartment by a mechanism involving ATG proteins, but not the conventional autophagic initiation

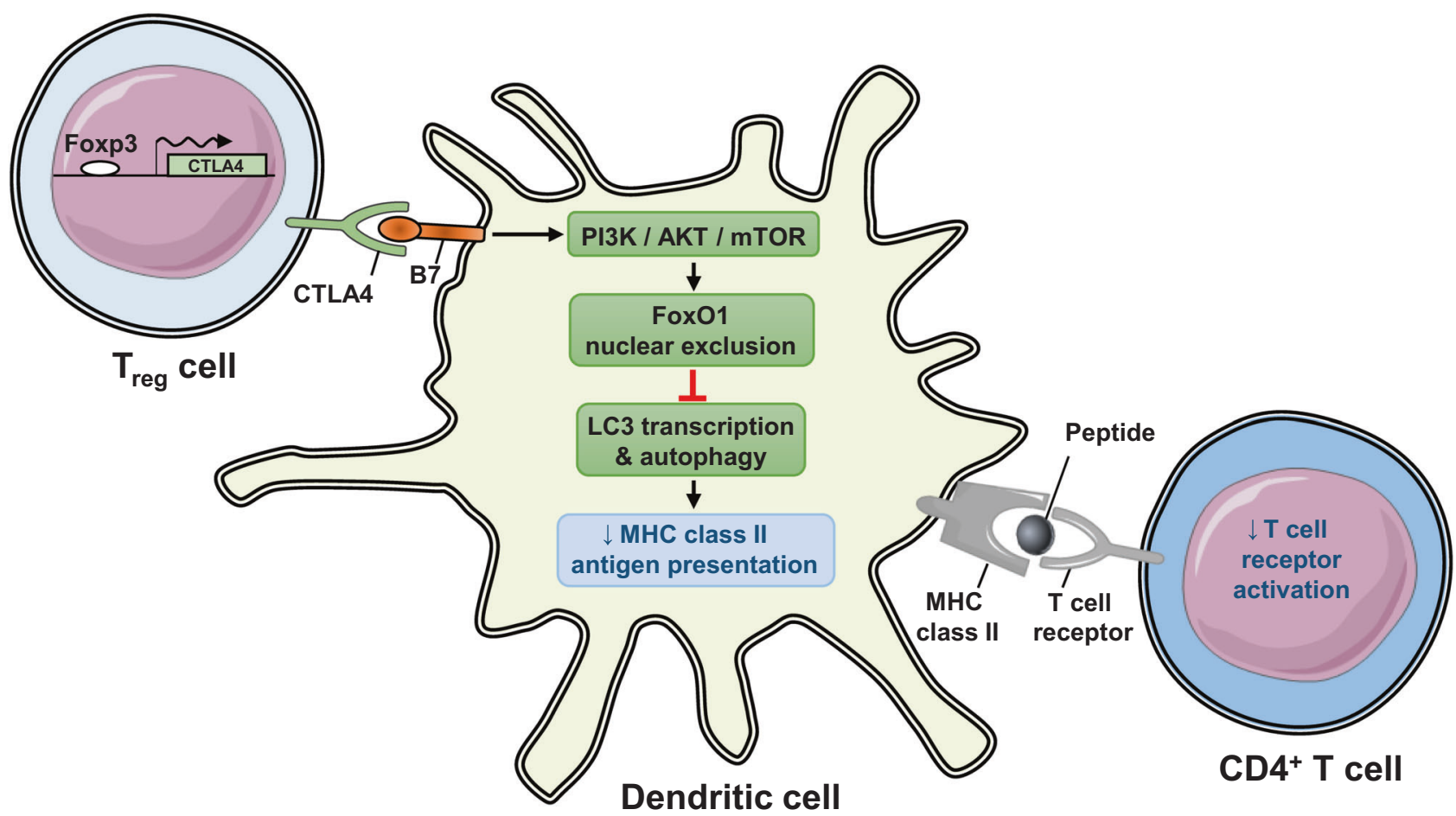

Fig. 5 Autophagy-mediated prevention of autoimmunity. Autophagy is required for DCs to be able to efficiently present peptides on MHC class II molecules. Regulatory $\mathrm{T}$ cells $\left(\mathrm{T}_{\text {regs }}\right)$ are important components in mechanisms of peripheral tolerance that control the development of autoimmune diseases. Inhibitory receptor CTLA4 on Foxp $3^{+} \mathrm{T}_{\text {regs }}$ suppresses the autophagy machinery in DCs, leading to decreased turnover of peptide-MHC class II complexes and decreased stimulation of peptide-specific $\mathrm{T}$ cell responses, resulting in the amelioration of autoimmune responses 
complex. The requirement for LAP to induce a DNAimmune complex-mediated IFN- $\alpha$ response in pDCs implies that this pathway could be involved in the pathogenesis of autoimmune diseases, where secreted type I IFNs contribute to disease progression [82].

\section{Concluding remarks}

Autophagy is a key cellular catabolic pathway that is utilized for multiple functions in different cell types. In the cells of the innate immune system, the special capabilities conferred by the autophagic pathway modulate dramatic changes seen during differentiation. Autophagy also modifies cell-specific functions such as antigen presentation, phagocytosis, and cytokine production. Autophagy can carry out responsibilities for microbial defence such as antigen presentation and production of antimicrobial peptides since pathogens can be directly digested in autophagolysosomes. Finally, the contributions of autophagy to the pathophysiology of inflammatory diseases are now recognized as a wide field for further investigation. Increasing awareness of the functional roles of autophagy in antimicrobial defence and an understanding of the modifications in these roles which can be achieved by particular bacteria and viruses promise a fruitful area of research and novel therapy approaches.

Acknowledgements The authors apologize to investigators whose contributions may not have been cited because of space limitations. The original work of the authors described here was supported by the Swiss National Science Foundation (31003A_173215 to S.Y. and 310030_166473 to H.U.S.) and the European Union Horizon 2020 Research and Innovation Program (Marie Sklodowska-Curie grant No. 642295; MEL-PLEX). N.G. and Z.F. are a PhD students of the Graduate School of Cellular and Biomedical Sciences of the University of Bern.

\section{Compliance with ethical standards}

Conflict of interest The authors declare that they have no conflict of interest.

Publisher's note: Springer Nature remains neutral with regard to jurisdictional claims in published maps and institutional affiliations.

\section{References}

1. Germic N, Frangez Z, Yousefi S, Simon HU. Regulation of the innate immune system by autophagy: Neutrophils, eosinophils, mast cells, NK cells. Cell Death Differ. (in press).

2. Mizushima N, Komatsu M. Autophagy: renovation of cells and tissues. Cell. 2011;147:728-41.

3. Yang Z, Klionsky DJ. Eaten alive: a history of macroautophagy. Nat Cell Biol. 2010;12:814-22.
4. Codogno P, Mehrpour M, Proikas-Cezanne T. Canonical and noncanonical autophagy: variations on a common theme of self-eating? Nat Rev Mol Cell Biol. 2011;13:7-12.

5. Kim JY, Zhao H, Martinez J, Doggett TA, Kolesnikov AV, Tang $\mathrm{PH}$, et al. Noncanonical autophagy promotes the visual cycle. Cell. 2013;154:365-76.

6. Niso-Santano M, Malik SA, Pietrocola F, Bravo-San Pedro JM, Mariño G, Cianfanelli V, et al. Unsaturated fatty acids induce noncanonical autophagy. EMBO J. 2015;34:1025-41.

7. Nichols BA, Bainton DF, Farquhar MG. Differentiation of monocytes. J Cell Biol. 1971;50:498-515.

8. Auffray C, Sieweke MH, Geissmann F. Blood monocytes: development, heterogeneity, and relationship with dendritic cells. Annu Rev Immunol. 2009;27:669-92.

9. Ginhoux F, Jung S. Monocytes and macrophages: developmental pathways and tissue homeostasis. Nat Rev Immunol. 2014;14:392-404.

10. Qian C, Cao X. Dendritic cells in the regulation of immunity and inflammation. Semin Immunol. 2018;35:3-11.

11. Merad M, Sathe P, Helft J, Miller J, Mortha A. The dendritic cell lineage: Ontogeny and function of dendritic cells and their subsets in the steady state and inflamed setting. Annu Rev Immunol. 2013;31:563-604.

12. Jacquel A, Obba S, Boyer L, Dufies M, Robert G, Gounon P, et al. Autophagy is required for CSF-1-induced macrophagic differentiation and acquisition of phagocytic functions. Blood. 2012;119:4527-31.

13. Zhang Y, Morgan MJ, Chen K, Choksi S, Liu ZG. Induction of autophagy is essential for monocyte-macrophage differentiation. Blood. 2012;119:2895-905.

14. Dorn BR, Dunn WA Jr, Progulske-Fox A. Bacterial interactions with the autophagic pathway. Cell Microbiol. 2002;4:1-10.

15. Ogawa M, Sasakawa C. Bacterial evasion of the autophagic defense system. Curr Opin Microbiol. 2006;9:62-68.

16. Nakagawa I, Amano A, Mizushima N, Yamamoto A, Yamaguchi $\mathrm{H}$, Kamimoto T, et al. Autophagy defends cells against invading group A Streptococcus. Science. 2004;306:1037-40.

17. Gutierrez MG, Master SS, Singh SB, Tylor GA, Colombo MI, Deretic V. Autophagy is a defense mechanism inhibiting BCG and Mycobacterium tuberculosis survival in infected macrophages. Cell. 2004;119:735-66.

18. Ogawa M, Yoshimori T, Suzuki T, Sagara H, Mizushima N, Sasakawa C. Escape of intracellular Shigella from autophagy. Science. 2005;307:727-31.

19. Birmingham CL, Smith AC, Bakowski MA, Yoshimori T, Brumell $\mathrm{JH}$. Autophagy controls Salmonella infection in response to damage to the Salmonella-containing vacuole. J Biol Chem. 2006;281:11374-83.

20. Sanjuan MA, Dillon CP, Tait SW, Moshiach S, Dorsey F, Connell $\mathrm{S}$, et al. Toll-like receptor signalling in macrophages links the autophagy pathway to phagocytosis. Nature. 2007;450:1253-7.

21. Xu Y, Jagannath C, Liu XD, Sharafkhaneh A, Kolodziejska KE, Eissa NT. Toll-like receptor 4 is a sensor for autophagy associated with innate immunity. Immunity. 2007;27:135-44.

22. Delgado MA, Elmaoued RA, Davis AS, Kyei G, Deretic V. Tolllike receptors control autophagy. EMBO J. 2008;27:1110-21.

23. Shi CS, Kehrl JH. MyD88 and Trif target Beclin 1 to trigger autophagy in macrophages. J Biol Chem. 2008;283:33175-82.

24. Shi CS, Kehrl JH. TRAF6 and A20 regulate lysine 63-linked ubiquitination of Beclin-1 to control TLR4-induced autophagy. Sci Signal. 2010;3:ra42.

25. Alvarez-Jiménez VD, Leyva-Paredes K, García-Martínez M, Vázquez-Flores L, García-Paredes VG, Campillo-Navarro M, et al. Extracellular vesicles released from Mycobacterium tuberculosisinfected neutrophils promote macrophage autophagy and decrease intracellular mycobacterial survival. Front Immunol. 2018;9:272. 
26. Travassos LH, Carneiro LA, Ramjeet M, Hussey S, Kim YG, Magalhães JG, et al. Nod1 and Nod2 direct autophagy by recruiting ATG16L1 to the plasma membrane at the site of bacterial entry. Nat Immunol. 2010;11:55-62.

27. Subauste CS, Andrade RM, Wessendarp M. CD40-TRAF6 and autophagy-dependent anti-microbial activity in macrophages. Autophagy. 2007;3:245-8.

28. Mills KR, Reginato M, Debnath J, Queenan B, Brugge JS. Tumor necrosis factor related apoptosis-inducing ligand (TRAIL) is required for induction of autophagy during lumen formation in vitro. Proc Natl Acad Sci. 2004;101:3438-43.

29. Park HJ, Lee SJ, Kim SH, Han J, Bae J, Kim SJ, et al. IL-10 inhibits the starvation induced autophagy in macrophages via class I phosphatidylinositol 3-kinase (PI3K) pathway. Mol Immunol. 2011;48:720-7.

30. Yuk JM, Shin DM, Lee HM, Yang CS, Jin HS, Kim KK, et al. Vitamin D3 induces autophagy in human monocytes/macrophages via cathelicidin. Cell Host Microbe. 2009;6:231-43.

31. Andrade RM, Wessendarp M, Gubbels MJ, Striepen B, Subauste CS. CD40 induces macrophage anti-Toxoplasma gondii activity by triggering autophagy-dependent fusion of pathogen-containing vacuoles and lysosomes. J Clin Invest. 2006;116:2366-77.

32. Ponpuak M, Davis AS, Roberts EA, Delgado MA, Dinkins C, Zhao Z, et al. Delivery of cytosolic components by autophagic adaptor protein p62 endows autophagosomes with unique antimicrobial properties. Immunity. 2010;32:329-41.

33. Sanjuan MA, Green DR. Eating for good health: linking autophagy and phagocytosis in host defense. Autophagy. 2008;4:607-11.

34. Bonilla DL, Bhattacharya A, Sha Y, Xu Y, Xiang Q, Kan A, et al. Autophagy regulates phagocytosis by modulating the expression of scavenger receptors. Immunity. 2013;39:537-47.

35. Biswas D, Qureshi OS, Lee WY, Croudace JE, Mura M, Lammas DA. ATP-induced autophagy is associated with rapid killing of intracellular mycobacteria within human monocytes/macrophages. BMC Immunol. 2008;9:35.

36. Zhao Z, Fux B, Goodwin M, Dunay IR, Strong D, Miller BC, et al. Autophagosome-independent essential function for the autophagy protein Atg5 in cellular immunity to intracellular pathogens. Cell Host Microbe. 2008;4:458-69.

37. Lima JG, de Freitas Vinhas C, Gomes IN, Azevedo CM, dos Santos RR, Vannier-Santos MA, et al. Phagocytosis is inhibited by autophagic induction in murine macrophages. Biochem Biophys Res Commun. 2011;405:604-9.

38. Martinez J, Malireddi RK, Lu Q, Cunha LD, Pelletier S, Gingras $\mathrm{S}$, et al. Molecular characterization of LC3-associated phagocytosis reveals distinct roles for Rubicon, NOX2 and autophagy proteins. Nat Cell Biol. 2015;17:893-906.

39. Heckmann BL, Boada-Romero E, Cunha LD, Magne J, Green DR. LC3-associated phagocytosis and inflammation. J Mol Biol. 2017;429:3561-76.

40. Saitoh T, Fujita N, Jang MH, Uematsu S, Yang BG, Satoh T, et al. Loss of the autophagy protein Atg16L1 enhances endotoxin induced IL-1 $\beta$ production. Nature. 2008;456:264-8.

41. Pu Q, Gan C, Li R, Li Y, Tan S, Li X, et al. Atg7 deficiency intensifies inflammasome activation and pyroptosis in Pseudomonas sepsis. J Immunol. 2017;198:3205-13.

42. Bechelli J, Vergara L, Smalley C, Buzhdygan TP, Bender S, Zhang W, et al. Atg 5 supports $R$. australis infection in macrophages in vitro and in vivo. Infect Immun. 2018;87:e00651-18.

43. Nakahira K, Haspel JA, Rathinam VA, Lee SJ, Dolinay T, Lam $\mathrm{HC}$, et al. Autophagy proteins regulate innate immune responses by inhibiting the release of mitochondrial DNA mediated by the NALP3 inflammasome. Nat Immunol. 2011;12:222-30.

44. Zhou R, Yazdi AS, Menu P, Tschopp J. A role for mitochondria in NLRP3 inflammasome activation. Nature. 2011;469:221-5.
45. Shi CS, Shenderov K, Huang NN, Kabat J, Abu-Asab M, Fitzgerald KA, et al. Activation of autophagy by inflammatory signals limits IL- $1 \beta$ production by targeting ubiquitinated inflammasomes for destruction. Nat Immunol. 2012;13:255-63.

46. Harris J, Hartman M, Roche C, Zeng SG, O'Shea A, Sharp FA, et al. Autophagy controls IL-1 $\beta$ secretion by targeting pro-IL-1 $\beta$ for degradation. J Biol Chem. 2011;286:9587-97.

47. Aflaki E, Moaven N, Borger DK, Lopez G, Westbroek W, Chae $\mathrm{JJ}$, et al. Lysosomal storage and impaired autophagy lead to inflammasome activation in Gaucher macrophages. Aging Cell. 2016;15:77-88.

48. Santeford A, Wiley LA, Park S, Bamba S, Nakamura R, Gdoura A, et al. Impaired autophagy in macrophages promotes inflammatory eye disease. Autophagy. 2016;12:1876-85.

49. Dai J, Zhang X, Li L, Chen H, Chai Y. Autophagy inhibition contributes to ROS-producing NLRP3-dependent inflammasome activation and cytokine secretion in high glucose-induced macrophages. Cell Physiol Biochem. 2017;43:247-56.

50. Liu K, Zhao E, Ilyas G, Lalazar G, Lin Y, Haseeb M, et al. Impaired macrophage autophagy increases the immune response in obese mice by promoting proinflammatory macrophage polarization. Autophagy. 2015;11:271-84.

51. Matta SK, Kumar D. AKT mediated glycolytic shift regulates autophagy in classically activated macrophages. Int $\mathrm{J}$ Biochem Cell Biol. 2015;66:121-33.

52. Stranks AJ, Hansen AL, Panse I, Mortensen M, Ferguson DJ, Puleston DJ, et al. Autophagy controls acquisition of aging features in macrophages. J Innate Immun. 2015;7:375-91.

53. Massey DC, Bredin F, Parkes M. Use of sirolimus (rapamycin) to treat refractory Crohn's disease. Gut. 2008;57:1294-6.

54. Münz C. Autophagy and antigen presentation. Cell Microbiol. 2006;8:891-8.

55. Mizushima N, Yamamoto A, Matsui M, Yoshimori T, Ohsumi Y. In vivo analysis of autophagy in response to nutrient starvation using transgenic mice expressing a fluorescent autophagosome marker. Mol Biol Cell. 2004;15:1101-11.

56. Nimmerjahn F, Milosevic S, Behrends U, Jaffee EM, Pardoll DM, Bornkamm GW, et al. Major histocompatibility complex class IIrestricted presentation of a cytosolic antigen by autophagy. Eur J Immunol. 2003;33:1250-9.

57. Dengjel J, Schoor O, Fischer R, Reich M, Kraus M, Müller M, et al. Autophagy promotes MHC class II presentation of peptides from intracellular source proteins. Proc Natl Acad Sci. 2005;102:7922-7.

58. Paludan C, Schmid D, Landthaler M, Vockerodt M, Kube D, Tuschl T, et al. Endogenous MHC class II processing of a viral nuclear antigen after autophagy. Science. 2005;307:593-6.

59. Zhou D, Li P, Lin Y, Lott JM, Hislop AD, Canaday DH, et al. Lamp-2a facilitates MHC class II presentation of cytoplasmic antigens. Immunity. 2005;22:571-81.

60. Schmid D, Pypaert M, Münz C. Antigen-loading compartments for major histocompatibility complex class II molecules continuously receive input from autophagosomes. Immunity. 2007;26:79-92.

61. Schmid D, Münz C. Innate and adaptive immunity through autophagy. Immunity. 2007;27:11-21.

62. Lee HK, Mattei LM, Steinberg BE, Alberts P, Lee YH, Chervonsky $\mathrm{A}$, et al. In vivo requirement for Atg5 in antigen presentation by dendritic cells. Immunity. 2010;32:227-39.

63. Romao S, Gasser N, Becker AC, Guhl B, Bajagic M, Vanoaica D, et al. Autophagy proteins stabilize pathogen-containing phagosomes for prolonged MHC II antigen processing. J Cell Biol. 2013;203:757-66.

64. Ma J, Becker C, Lowell CA, Underhill DM. Dectin-1-triggered recruitment of light chain 3 protein to phagosomes facilitates major histocompatibility complex class II presentation of fungalderived antigens. J Biol Chem. 2012;287:34149-56. 
65. Cooney R, Baker J, Brain O, Danis B, Pichulik T, Allan P, et al. NOD2 stimulation induces autophagy in dendritic cells influencing bacterial handling and antigen presentation. Nat Med. 2010;16:90-7.

66. Ireland JM, Unanue ER. Autophagy in antigen-presenting cells results in presentation of citrullinated peptides to CD4 T cells. J Exp Med. 2011;208:2625-32.

67. Jagannath C, Lindsey DR, Dhandayuthapani S, Xu Y, Hunter RL Jr, Eissa NT. Autophagy enhances the efficacy of BCG vaccine by increasing peptide presentation in mouse dendritic cells. Nat Med. 2009;15:267-76.

68. Ravindran R, Khan N, Nakaya HI, Li S, Loebbermann J, Maddur MS, et al. Vaccine activation of the nutrient sensor GCN2 in dendritic cells enhances antigen presentation. Science. 2014;343:313-7.

69. Saini NK, Baena A, Ng TW, Venkataswamy MM, Kennedy SC, Kunnath-Velayudhan S, et al. Suppression of autophagy and antigen presentation by Mycobacterium tuberculosis PE_PGRS47. Nat Microbiol. 2016;1:16133.

70. Loi M, Müller A, Steinbach K, Niven J, Barreira da Silva R, Paul $\mathrm{P}$, et al. Macroautophagy proteins control MHC class I levels on dendritic cells and shape anti-viral CD8 $+\mathrm{T}$ cell responses. Cell Rep. 2016;15:1076-87.

71. Tey SK, Khanna R. Autophagy mediates transporter associated with antigen processing-independent presentation of viral epitopes through MHC class I pathway. Blood. 2012;120:994-1004.

72. Budida R, Stankov MV, Döhner K, Buch A, Panayotova-Dimitrova D, Tappe KA, et al. Herpes simplex virus 1 interferes with autophagy of murine dendritic cells and impairs their ability to stimulate CD8+ T lymphocytes. Eur J Immunol. 2017;47:1819-34.

73. Lee HK, Lund JM, Ramanathan B, Mizushima N, Iwasaki A. Autophagy-dependent viral recognition by plasmacytoid dendritic cells. Science. 2007;315:1398-401.

74. Manuse MJ, Briggs CM, Parks GD. Replication-independent activation of human plasmacytoid dendritic cells by the paramyxovirus SV5 requires TLR7 and autophagy pathways. Virology. 2010;405:383-9.

75. Severa M, Giacomini E, Gafa V, Anastasiadou E, Rizzo F, Corazzari M, et al. EBV stimulates TLR- and autophagy-dependent pathways and impairs maturation in plasmacytoid dendritic cells: implications for viral immune escape. Eur $\mathrm{J}$ Immunol. 2013;43:147-58.

76. Zang F, Chen Y, Lin Z, Cai Z, Yu L, Xu F, et al. Autophagy is involved in regulating the immune response of dendritic cells to influenza A (H1N1) pdm09 infection. Immunology. 2016;148:5669.

77. Lupfer C, Thomas PG, Anand PK, Vogel P, Milasta S, Martinez J, et al. Receptor interacting protein kinase 2-mediated mitophagy regulates inflammasome activation during virus infection. Nat Immunol. 2013;14:480-8.

78. Blanchet FP, Moris A, Nikolic DS, Lehmann M, Cardinaud S, Stalder R, et al. Human immunodeficiency virus-1 inhibition of immunoamphisomes in dendritic cells impairs early innate and adaptive immune responses. Immunity. 2010;32:654-69.

79. Reed M, Morris SH, Jang S, Mukherjee S, Yue Z, Lukacs NW. Autophagy-inducing protein beclin-1 in dendritic cells regulates CD4 $\mathrm{T}$ cell responses and disease severity during respiratory syncytial virus infection. J Immunol. 2013;191:2526-37.

80. Baghdadi M, Yoneda A, Yamashina T, Nagao H, Komohara Y, Nagai S, et al. TIM-4 glycoprotein-mediated degradation of dying tumor cells by autophagy leads to reduced antigen presentation and increased immune tolerance. Immunity. 2013;39:1070-81.

81. Alissafi T, Banos A, Boon L, Sparwasser T, Ghigo A, Wing K, et al. Tregs restrain dendritic cell autophagy to ameliorate autoimmunity. J Clin Invest. 2017;127:2789-804.

82. Henault J, Martinez J, Riggs JM, Tian J, Mehta P, Clarke L, et al. Noncanonical autophagy is required for type I interferon secretion in response to DNA-immune complexes. Immunity. 2012;37:98697. 